\title{
¿CÓMO REPRESENTAR A LOS REPRESORES EN UN SITIO DE MEMORIA? EL CASO DEL PARQUE POR LA PAZ VILLA GRIMALDI*
}

\author{
HOW TO REPRESENT HUMAN RIGHTS ABUSERS IN A SITE OF \\ MEMORY? THE CASE OF THE PARQUE POR LA PAZ VILLA GRIMALDI
}

\section{DANIEL REBOLLEDO HERNÁNDEZ ${ }^{\star *}$, OMAR SAGREDO MAZUELA***}

Resumen: El artículo analiza la representación de los violadores de derechos humanos en el sitio de memoria Parque por la Paz Villa Grimaldi, mediante tres dimensiones: relato histórico y pedagógico, museografía y opiniones de los visitantes. Si bien la representación de los perpetradores está condicionada por el mandato de reparación simbólica de sobrevivientes y familiares del sitio, la investigación evidencia, por una parte, que, con la integración de nuevos dispositivos (audio-guías) y elementos expositivos sobre la historia del lugar, la figuración de estos sujetos aparece gradualmente, permitiendo precisar distinciones (particularmente, entre guardias y torturadores) y, por otra parte, que los visitantes demandan crecientemente más información sobre represores.

Palabras clave: Represor, sitio de memoria, museo, representación, derechos humanos.

Aвstract: This paper analyses the representation of perpetrators of human rights violations in the site of memory Parque por la Paz Villa Grimaldi. It focuses on three dimensions: historical and pedagogical narrative, museography, and visitors' opinions. Originally, references to perpetrators were scarce because the site's mission concentrated on reparations to survivors and victims' relatives. Nevertheless, as this study argues, the representation of perpetrators has gradually increased due to the use of audioguides and other museographic devices, which have diversified the perpetrators' image by marking some distinctions between torturers and guards. Finally, this study also shows that visitors are increasingly demanding more information about perpetrators.

KEYwORDs: Human rights abusers, site of memory, museum, depictions, human rights.

Recibido: 01.02.19. Aceptado: 9.03.20.

\footnotetext{
* Este artículo es resultado de un estudio de interés personal de los autores, y no representa, necesariamente, el pensamiento del Directorio de la Corporación Parque por la Paz Villa Grimaldi (CPPVG), ni de los trabajadores que la conforman. Así mismo, los autores señalan que para esta investigación se utilizó la colección testimonial del Archivo Oral de la CPPVG, y declaran que los resultados de la misma son de responsabilidad propia y en nada comprometen a esta institución.

** Maestrando en Planificación Territorial y Gestión Ambiental. Corporación Parque por la Paz Villa Grimaldi, Santiago, Chile. Correo electrónico: daniel.rebolledo@villagrimaldi.cl. Orcid: http:// orcid.org/0000-0001-6026-716X.

${ }_{* * \star}$ Magíster en Estudios Internacionales. Corporación Parque por la Paz Villa Grimaldi, Santiago, Chile. Correo electrónico: omar.sagredo@villagrimaldi.cl. Orcid: http://orcid.org/0000-0003-4481-4260.
} 


\section{INTRODUCCIÓN}

E L PARque por la Paz Villa Grimaldi (PPVG) es un sitio de memoria erigido sobre los vestigios de uno de los principales centros secretos de detención, tortura y exterminio (CDTE) de la dictadura cívico-militar chilena (1973-1990). Denominado “Cuartel Terranova” por los organismos represores, el recinto operó entre 1974 y $1978^{1}$, y se estima que, aproximadamente, cuatro mil quinientas personas estuvieron secuestradas allí, de las cuales doscientas cuarenta y una fueron asesinadas o hechas desaparecer.

En el marco de la estrategia de ocultamiento de la dictadura, a través de la desaparición de los recintos represivos (Santos-Herceg, 2016a, pp. 257258), Villa Grimaldi comenzó a ser demolida a fines de la década de 1980. Sin embargo, la Asamblea Permanente por los Derechos Humanos Peñalolén-La Reina, un colectivo de la sociedad civil compuesto por vecinos del sitio, ex detenidos, familiares de víctimas y comunidades cristianas, luego de una extensa campaña de demarcación y denuncia, consiguió recuperar el sitio en 1993, logrando que el Estado lo expropiara. En 1994, el Servicio de Vivienda y Urbanización convocó propuestas para el proyecto Villa Grimaldi, resultando seleccionada la alternativa del Parque por la $\mathrm{Paz}^{2}$, el cual se inauguró en 1997. El esquema de representación de la memoria del pasado reciente del sitio se determinó como un espacio abierto a la conmemoración de las víctimas y la reparación simbólica de sobrevivientes y familiares. De acuerdo a esta configuración, así como a definiciones posteriores acerca del sentido de los "sitios de memoria", la figura de los represores no era contemplada como un elemento a considerar en la narrativa o los soportes gráficos de apoyo.

Sin embargo, actualmente, en el país existen planteamientos críticos sobre el campo del patrimonio cultural y la historia reciente que permiten vislumbrar oportunidades de debate importantes sobre la presencia de la figura de los victimarios en los sitios de memoria. Por una parte, el cuestionamiento al uso de definiciones tradicionales de patrimonio aplicadas a los ex CDTE (Elgueta, 2018) y la proposición de conceptualizaciones propias, derivadas del campo del "patrimonio disonante" (Tunbridge y Ashworh, 1996), tales como "patrimonio de la atrocidad" (Alegría, 2011), "patrimonio hostil" (Aguilera, 2019) y "patrimonio de la memoria de las violaciones

\footnotetext{
${ }^{1}$ Siendo el espacio de funcionamiento de la Brigada de Inteligencia Metropolitana (BIM) de la Dirección de Inteligencia Nacional (DINA) (Salazar, 2013, p. 97).

${ }^{2}$ Para profundizar en el proceso de creación del Parque por la Paz Villa Grimaldi, revisar Dalla Porta et al. 2017, pp. 69-73.
} 
a los derechos humanos" (Seguel, 2017). El objetivo tras aquellos planteamientos es caracterizar estos lugares como sitios de conciencia, es decir, espacios que no solo conmemoran y reparan a las víctimas, sino que, además, aportan a reinterpretar la historia oficial y fomentar el diálogo sobre temas sociales apremiantes actuales (Coalition of Sites of Conscience, 2018). Por otra parte, el avance y profundización de la discusión acerca de la representación de la dictadura y los represores en el espacio público nacional (Jara y Aguilera, 2017), debate que involucra interrogantes tales como la singularidad de Pinochet y su régimen en la historia del país (Basaure, 2018) y la posibilidad de abordar la distinción entre civiles y militares como agentes de la represión dictatorial (Sepúlveda, 2016). De entre estos dilemas, la mayor controversia nacional se ha situado en la representación de los victimarios en los museos históricos (Estefane, 2019; Mallea y Meirovich, 2019).

En consideración de lo anterior, el presente artículo aborda la situación del PPVG respecto de la representación de perpetradores en tres dimensiones: relato, museografía e interés de las audiencias. En ausencia de literatura nacional sobre el tema, se sostiene que la trayectoria del PPVG en esta materia ha seguido los planteamientos de Salvi $(2016,2017)$, considerando la representación de victimarios como un esfuerzo de denuncia y condena pública que permite distinguir las especificidades del aparato represor, y de Gryglewski (2017), en tanto oportunidad de aprendizaje e investigación para generaciones post-dictadura.

\section{NATURALEZA SIMBÓLICA Y PROYECTO MUSEO DEL PARQUE POR LA PAZ VILLA GRIMALDI}

A partir de la articulación de simbolismos relativos a la esperanza, solidaridad y memoria ${ }^{3}$, y sobre el esquema simbólico de dos ejes, que representaban la "vida" y la "muerte" (el primero asociado al actual acceso de visitantes y el segundo, relacionado tanto con el ingreso de prisioneros secuestrados, como con su salida hacia la muerte y/o desaparición), el PPVG fue inaugu-

\footnotetext{
${ }^{3}$ El equipo de arquitectos buscó "marcar" el sitio en el entramado urbano y confeccionar un esquema interno a través de reconstrucciones emblemáticas. De acuerdo con Ana Cristina Torrealba, integrante del equipo de arquitectos del proyecto Parque: “(...) la postura que imperó, que tomó fuerza, fue la resignificación. Y eran muchos los sobrevivientes que habían estado fuera y habían conocido Auschwitz y otros lugares y hablaban de lo morboso que era y de lo cruel que era" (fragmento del testimonio de Torrealba para el Archivo Oral de Villa Grimaldi).
} 
rado como el primer sitio de memoria en Chile ${ }^{4}$. Siendo, además, el primer ex CDTE recuperado en América Latina, el proceso de organización del lugar fue complejo y polémico. Existieron propuestas que apuntaban tanto a no intervenir el sitio como a reconstruir el ex Cuartel Terranova (Lazzara, 2003, p. 131).

Si bien la "vida" era el elemento convocante del sitio en tanto espacio de resignificación, la "muerte" tuvo expresión en el segundo eje, que comenzaba en el portón original y finalizaba en los cimientos de la destruida torre, principal espacio ligado, según el relato de las y los sobrevivientes, a la desaparición forzada. Sin embargo, las características principales del proyecto destacaban mayoritariamente elementos relativos a la "vida", la reparación y la paz, clausurando material y simbólicamente la "muerte" a través del cierre permanente del portón de ingreso original. En aquel contexto, en términos de narrativa y visualidad, la represión y sus agentes no contaban, por tanto, con un lugar o relato de representación. Sobre esto último es importante mencionar que un amplio sector de víctimas y familiares, representados en funciones directivas del PPVG, se opusieron a que el sitio fuera denominado "Cuartel Terranova" en documentos o soportes desarrollados por la institución, señalando que ese término corresponde al "lenguaje de los represores".

Posteriormente, en consideración de exigencias tanto de un sector de la comunidad de ex presos y familiares de víctimas del sitio, como de una serie de descubrimientos de vestigios, el Parque por la Paz experimentó una serie de modificaciones ${ }^{6}$. En materia de representación, en el año 2000 se incorporaron réplicas de una de las celdas y de la torre, simbolizando un "cierre" material al relato del "eje de la muerte".

\footnotetext{
${ }^{4}$ Es necesario señalar que el uso del concepto "sitio de memoria" es posterior. Inicialmente, se utilizaba términos como "centro" y "organismo". Actualmente, el espacio es un museo de sitio, declarado en 2004 como Monumento Nacional, en la categoría de Monumento Histórico. Recibe anualmente a más de veinte mil visitantes y entre sus actividades regulares se destacan la realización de acciones de conmemoración, instancias artísticas y culturales de promoción de los derechos humanos y ejercicios de educación a partir de los principios de la pedagogía de la memoria.

${ }^{5}$ Una lectura problematizada acerca del "lenguaje de los represores" utilizado por algunas víctimas del terrorismo de Estado se encuentra en Mallol (2009, pp. 41-42). El autor cuestiona el uso acrítico de determinados conceptos, tales como "colaborador", "quebrado" y "héroe", señalando que corresponden a la estrategia de los victimarios de responsabilizar a las víctimas por su destino.

${ }^{6}$ En 2007, se logró recuperar una fracción de las originales escalinatas de la casona, así como una explanada de adoquines. Estas revelaciones tensionaron uno de los principios sobre lo que se sustentó el proyecto Parque por la Paz: la imposibilidad de hallar vestigios. (Aguilera, 2011b, p. 71).
} 
El carácter simbólico del sitio permitió, por un lado, ampliar las formas de resignificar lo ocurrido, pero, por otro lado, acentuó la complejidad de las decisiones acerca de cómo narrar las atrocidades cometidas (Alegría, 2010, p. 96). En este sentido, el proyecto Museo Villa Grimaldi, desarrollado entre los años 2010 y 2011, debió afrontar el desafío de transitar desde un espacio de "paz y sanación", a un museo de sitio con perspectiva crítica, en el que los vestigios se presentaran jerárquicamente por sobre lo simbólico, y se apuntara a la generación de conciencia sobre situaciones vejatorias del presente (Aguilera, 2011b, pp. 104-105). Uno de los pilares de esta iniciativa fue la contextualización del terrorismo de Estado, centrado en la experiencia de Villa Grimaldi, relacionando sus efectos con la calidad de la democracia del presente mediante estrategias de pedagogía de la memoria. (Romero, 2011, pp. 13-14).

\section{LA REPRESENTACIÓN DE LOS VICTIMARIOS EN EL RELATO}

\section{a) Audio-guías}

El audio-guía ${ }^{7}$, un soporte elaborado entre 2009 y 2010 en el marco del proyecto "visita auto-guiada", presenta, inicialmente, a los perpetradores a partir de referencias a la trayectoria histórica de Villa Grimaldi.

Hacia fines de 1973 la Dirección de Inteligencia Nacional (DINA), dirigida por Manuel Contreras Sepúlveda, presionó directamente a Vasallo ${ }^{8}$ y ocupó Villa Grimaldi. En 1975, el Ministerio de Vivienda la expropió y cinco años más tarde, en 1980, la vendió a la Central Nacional de Informaciones (CNI), organismo sucesor de la DINA. Este dato es importante, porque, años más tarde esta venta sirvió para desmentir la versión de Manuel Contreras ante los Tribunales de Justicia en la que negaba toda vinculación entre la DINA y Villa Grimaldi.

El 21 de septiembre de 1987, el último director de la CNI, el general Hugo Salas Wenzel, vendió la propiedad de manera fraudulenta a la Sociedad Constructora E.G.P.T. Ltda., empresa conformada por sus propios familiares. (Pista 2: Maqueta).

${ }^{7}$ Es posible revisar de manera íntegra el audio-guía del PPVG en el siguiente link: http://villagrimaldi.cl/visite-el-parque/audioguias/ Todas las referencias que se mencionan a continuación corresponden a extractos de este.

${ }^{8}$ Dueño original del sitio. 
Otras referencias similares, respecto del funcionamiento de la DINA, se presentan en los hitos "Torre" y "Sala de la memoria", en relación a la disposición de las celdas y las acciones de falsificación de documentos, respectivamente. En el punto "Piscina", se aborda un hecho importante respecto de la conducta de los represores, en referencia a cómo los agentes utilizaban algunas zonas de Villa Grimaldi como lugares de esparcimiento.

A pesar de que durante su etapa como Cuartel Terranova la Villa Grimaldi era ocupada para esconder y torturar, conservaba cierto encanto de su pasado como casa familiar. Por eso es que paradojalmente se convirtió en un lugar de descanso para los agentes de la DINA y sus familiares. Algunos sobrevivientes que estuvieron en la Torre, recuerdan que en tardes de verano escuchaban a familias de paseo y niños bañándose en la piscina. Algunos cuentan que se oían carcajadas, música y sonidos de vasos chocando en brindis. Los agentes de la DINA estimaban que el mismo cuartel donde torturaban y asesinaban era un buen lugar para descansar con sus familias en sus días libres. (Pista 12: Piscina).

Este comportamiento de los perpetradores permite el acercamiento a dos asuntos. Por un lado, el modo en que los agentes se relacionaban con el CDTE, manifestándose lo que el sitio representaba para ellos: un lugar totalmente apropiado, fuera de cualquier control o delimitación normativa externa (Santos-Herceg, 2016b, p. 168). Por otro lado, se hace referencia a una arista de la psicología de los victimarios respecto de la forma en que las acciones represivas parecen estar desprovistas de connotaciones negativas, asumiendo el convencimiento de su pretendida condición de gestores históricos positivos para el desarrollo del país?.

Una última referencia a los represores en el audio-guía es la descripción del caso del soldado Carlos Carrasco Matus, quien, habiendo pertenecido a la DINA en condición de guardia, fue secuestrado por otros agentes, y luego, hecho desaparecer. Diversos testimonios hablan de Carrasco como un guardia distinto que no solo presentaba un trato humanitario, sino que los protegía de las torturas. El siguiente texto corresponde a la pista extraordinaria dedicada en el audio-guía al caso de Carrasco:

Carlos Alberto Carrasco Matus, fue un joven ex militante del Movimiento de Acción Popular Unitaria (MAPU), que en 1973 debió cumplir con

\footnotetext{
${ }^{9}$ Esta interpretación se vincula con el desarrollo de la memoria emblemática relacionada con la salvación del país (Stern, 1999, p. 15).
} 
el servicio militar obligatorio (...) siendo destinado como guardia a los centros secretos de Cuatro Álamos y Cuartel Terranova. El 14 de marzo de 1975, mientras Carlos Carrasco hacía uso de sus vacaciones, fue detenido en su casa por dos agentes, quienes se identificaron como funcionarios de la DINA. (...) De acuerdo a testimonios de varios ex prisioneros, 'El Mauro', como era conocido Carrasco Matus, era una 'excepción' entre los guardias, ya que siempre tuvo una actitud humanitaria y de ayuda hacia ellos. El testimonio de un sobreviviente de Villa Grimaldi y que también pasó por Tres Álamos, señala que tras los interrogatorios lo dejan en la torre a cargo de un equipo de 'ablandamiento', encontrándose aquí con un tipo alto, que le dice que no le hará nada, pero que si le preguntan otros agentes, se queje simulando que había sido golpeado. Este mismo sobreviviente, es llevado a Cuatro Âlamos, donde también se encuentra con "el Mauro". Sin embargo, después del 26 de febrero, no se le volvió a ver en ese recinto. (...) Otro testimoniante señala que el Mauro fue engrillado y colgado al Ombú. Luego se obliga a todos los guardias y agentes a pegarle con una cadena, hasta matarlo, como medida de escarmiento. Carlos Carrasco Matus, es en la actualidad un detenido desaparecido. (Pista 17: Ombú. El caso del conscripto Carlos Alberto Carrasco Matus).

El caso de Carrasco ha sido incorporado a la narrativa de Villa Grimaldi como una excepción entre el común de los guardias. Reconociendo las distinciones existentes entre los diferentes agentes que operaban en los CDTE (tal como se señaló en Calveiro, 2006, y Sepúlveda, 2016), la condición de víctima del terrorismo de Estado de Carrasco ha sido ratificada más allá del relato testimonial de algunos sobrevivientes, a través de la inclusión de su historia en la Sala de la Memoria ${ }^{10}$ y en su permanencia, luego de la más reciente actualización, en la nómina de ejecutados/as políticos/as y detenidos/as desaparecidos/as de Villa Grimaldi ${ }^{11}$.

La situación de Carrasco, no obstante, es única en el relato y la museografía del sitio, por lo que su inclusión podría ser interpretada como una

\footnotetext{
${ }^{10}$ La vitrina de la Sala de Memoria que corresponde a Carrasco contiene una camisa (la misma que él utilizó el día en que la DINA lo detuvo en su casa), una carta escrita por Carlos a su madre en 1961, un dibujo que representa el Ombú de Villa Grimaldi, un distintivo de cabo primero, un parche de infantería del Regimiento número 1 de Buin y siete fotografías de distintos momentos de su vida.

${ }^{11}$ En el reciente libro 20 años sitio de Memoria Parque por la Paz Villa Grimaldi, el caso de Carlos Carrasco es descrito de la siguiente forma: "Fue designado para desempeñarse como guardia de los centros de detención de Tres y Cuatro Álamos. Fue secuestrado por agentes de la DINA desde su casa, luego de detectar su trato humanitario con los prisioneros y sus labores de entrega de información hacia familiares y otros militantes" (Dalla Porta et al., 2017, p. 44).
} 
expresión de "memoria ejemplificadora" (Marchesi, 2018, p. 285), en tanto permite obtener lecciones sobre el pasado y representar situaciones generales acerca de la realidad que experimentaron los conscriptos de las Fuerzas Armadas durante el gobierno dictatorial ${ }^{12}$.

\section{b) Guión de recorridos pedagógicos}

Durante el año 2017, el Área Educación del Parque por la Paz elaboró un guion para sus recorridos pedagógicos ${ }^{13}$. En este documento, las primeras alusiones a los represores se manifiestan de manera similar al audio-guía, es decir, revisando las características de los organismos de seguridad.

(...) la Junta Militar decidió reprimir de manera secreta, para ocultar los crímenes de lesa humanidad que estaban cometiendo. Así, la Junta creó una policía secreta específica para estos fines, que llamó Dirección de Inteligencia Nacional, DINA. Fue dirigida por Manuel Contreras. Ellos ya no se vestían de uniforme como los militares los primeros días, sino como cualquier civil, y como parte de su estrategia, esta policía secreta comenzó a buscar lugares propicios para ejercer esta represión. La DINA ocupó mediante amenaza este lugar, y en la casona que vemos ahí (señalar la casa en la maqueta), que antes era restaurant, instaló sus oficinas. Allí operó la Brigada de Inteligencia Metropolitana (BIM), (...). (Hito 2: Maqueta).

A diferencia del audio-guía, el guion de los recorridos pedagógicos se extiende en algunas características de la DINA, y en adición, de sus integrantes. Referencias importantes, en este sentido, para comprender detalles del terrorismo de Estado a partir del comportamiento de los agentes, es su forma de vestir y las estrategias de elección de los CDTE. Una segunda alusión se encuentra en el hito "Muro de los Nombres", lugar donde la narrativa aborda el proceso de justicia y la no colaboración de los ex agentes.

\footnotetext{
${ }^{12}$ Para profundizar en la situación de los conscriptos de la dictadura, se recomienda el trabajo de Bustamante (2007, pp. 325-330).

${ }^{13} \mathrm{El}$ guion de los recorridos pedagógicos del Área Educación del PPVG es un documento de uso interno. Sin embargo, los autores, en tanto integrantes del Área Museo de esta institución, pueden gestionar el acceso a este documento a quienes lo soliciten, previa evaluación del requerimiento. Todas las referencias que se mencionan a continuación corresponden a extractos de este.
} 
Aquellas personas que perpetraron estos crímenes han entregado muy poca información, pues entre ellos realizaron un pacto de silencio que mantienen hasta el día de hoy. La justicia y las condenas para los responsables han sido muy escasas y han contribuido a que aún hoy muchos familiares no sepan dónde están los cuerpos de sus seres queridos, ni cuáles fueron las circunstancias de secuestro y muerte. Esta situación de impunidad es una de las principales razones por las que ha sido tan difícil reconstruir la historia de lo que sucedió en el Cuartel Terranova. (Hito 4: Muro de los Nombres)

En el hito "Sala de la Memoria", se aborda el uso de este espacio por parte de la DINA en referencia a la falsificación de documentos, del mismo modo en que esta situación es presentada en el relato del audio-guía.

\section{LA REPRESENTACIÓN DE LOS VICTIMARIOS EN LA MUSEOGRAFÍA}

La museografía del Parque por la Paz comprende el conjunto de elementos introducidos con el objetivo de mejorar/aportar, en términos de contenidos, la experiencia de visita al sitio. Estos soportes son de variado tipo (dibujos, folletos, paneles informativos, reproducciones, módulos interactivos), y han sido incorporados y/o coordinados, principalmente, por el Área Museo de la Corporación, de acuerdo a los principios de museo de sitio y gestión patrimonial. En este punto, el primer elemento de representación de perpetradores incorporado al sitio fue un dibujo realizado en 2005 por el ex detenido de Villa Grimaldi, Miguel Montecinos, dispuesto al interior de la Torre. La imagen 1 muestra este dibujo, en el que se observa a tres agentes que personifican las distintas funciones represivas ejercidas en el lugar, las cuales coinciden con la identificación realizada por Calveiro (2006, p. 37) respecto de los CDTE en Argentina: torturador, agente de inteligencia y guardia. 


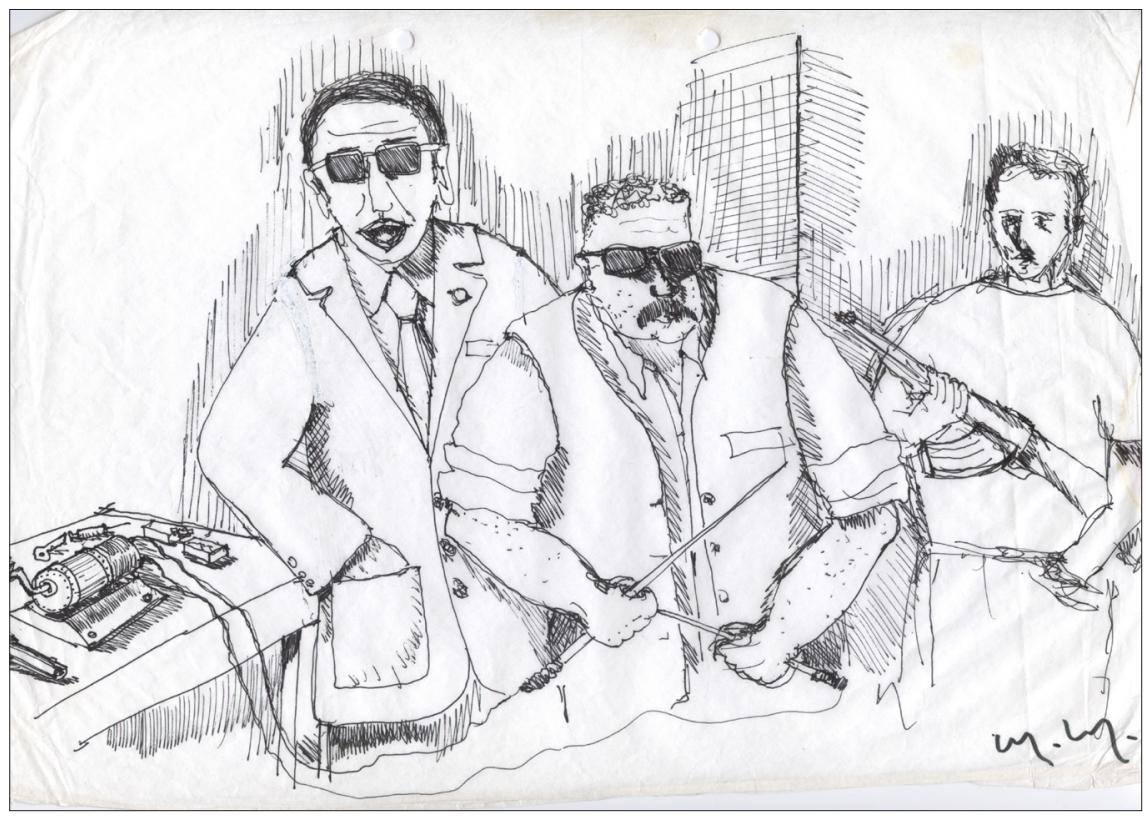

Figura 1. Dibujo de Miguel Montecinos.

El primer personaje de la imagen representa a aquellos agentes que ejercían el rol de interrogadores, quienes supervisaban y coordinaban las acciones de tortura. También, este sujeto puede personificar a los médicos que participaban de la tortura, controlando el estado de las/os detenidas/os, colaborando con los represores, permitiendo un acercamiento al papel de los civiles en los organismos represivos. El segundo personaje (ubicado al centro de la imagen), representa al torturador, un sujeto robusto que sostiene en sus manos el aparato con que se aplicaban descargas eléctricas a las/ os detenidas/os. Finalmente, el tercer agente, quien porta un arma, simboliza a un guardia. Visiblemente más joven que los demás, este individuo de vigilancia y cuidado de las/os secuestradas/os. Por cierto, entre los testimonios de sobrevivientes de Villa Grimaldi, esta distinción entre torturadores y guardias aparece frecuentemente mencionada ${ }^{14}$.

${ }^{14}$ Algunos fragmentos de testimonios de ex detenidos de Villa Grimaldi, extraídos desde el Archivo Oral del Parque por la Paz, ratifican esta distinción: “(...) a veces llegaba la guardia simpática que nos traía algún cigarrillo, y conversamos (sic) hasta tarde en la noche. (...)”; " había guardias que no tenían esta misma actitud. (...), todavía conservaban una cierta humanidad y de buen trato. Por lo menos el trato respetuoso, en la medida que podían. Incluso hay uno que fue bastante bueno con una que estaba esperando un hijo y como que trataba un poco de protegerla." 
Otra representación de los perpetradores es el organigrama de la DINA dispuesto en la Torre en 2012. En la imagen 2, se observa este soporte de $170 \mathrm{~cm}$ por $120 \mathrm{~cm}$, que muestra la composición del organismo represivo,

\section{ESTRUCTURA REPRESIVA DIRECCIÓN OE INTELIGENCIA NACIONAL (DINA) EN VILLA GRIMALDI}

JUNTA MILITAR DE GOBIERND (CREADA POR DECRETO LEY N 1 \%EL 11 DE SEPTIEMBRE DE 1973)

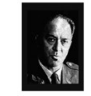

GUSTAVO LEGG GUIZMAN

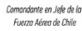

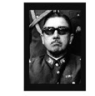

AUGUSTO PINOCHET UGARTE

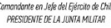

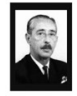

JOSÉ TORBBIO MERINO

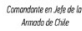

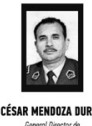

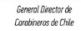

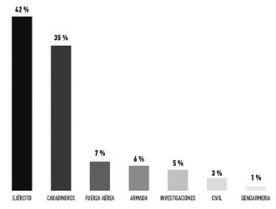

RAMAS DE LAS FFAA.Y Y CARABINEROS, PORCENTAJE DE ACCIONAR REPRESIVO

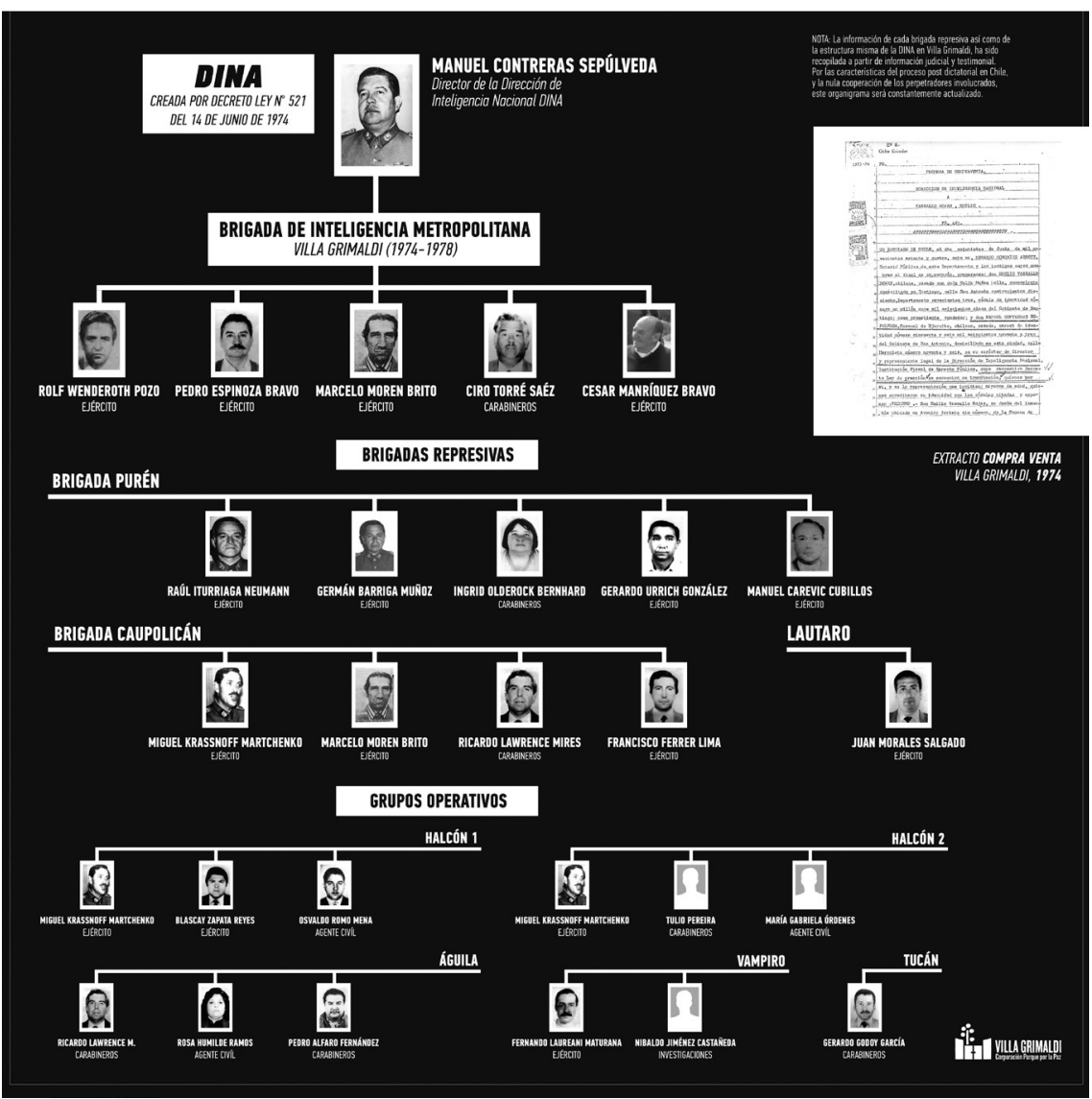

Figura 2. Organigrama DINA. 
comenzando con la Junta Militar. Se presentan las brigadas (Purén y Caupolicán), los grupos operativos y a los integrantes que las conformaban. Se incluye también el porcentaje de efectivos por rama de las Fuerzas Armadas, en relación a su participación en el accionar represivo. La confección del organigrama se realizó a partir de antecedentes judiciales e información proporcionada por la Brigada de Derechos Humanos de la Policía de Investigaciones (PDI), y se decidió incluir, únicamente, a aquellos agentes que habían sido condenados penalmente.

En concreto, esta imagen fue pensada con dos objetivos: reconocer a los principales agentes, (distinguiendo entre los distintos grupos operativos y reconociendo a civiles) y abordar el desarrollo de la justicia en términos histórico y penal, coincidiendo con los fines propuestos por Salvi (2017) respecto de cómo se ha representado a los represores en la Ex ESMA: "gesto político de denuncia y gesto institucional de enjuiciamiento" (p. 44). El primer objetivo, que constituye una acción de denuncia, puede lograrse de manera directa, con la observación de rostros, nombres y funciones, lo que constituye una acción significativa considerando que en ninguno de los informes de verdad realizados por el Estado se ha mencionado el nombre de los victimarios (Collins, 2013, p. 103). El segundo fin, sin embargo, requiere de mediación, es decir, de una acción de interpretación que logre relacionar nombre, función y situación judicial. Esta mediación puede concretarse mediante los sistemas de atención de público y trabajo con los visitantes (uso del audio-guía y participación en recorridos pedagógicos, respectivamente), en un esquema de generación de experiencias en el que los sujetos interpretan la historia y elaboran sus propias preguntas y reflexiones ${ }^{15}$.

La selección de la Torre como espacio de disposición de ambos elementos se adoptó considerando los resultados del estudio de público realizado entre 2011 y $2012^{16}$. Dos asuntos preliminares para esta decisión fueron: la condición cerrada que presenta la Torre (lo que permite separar el interior de ésta del resto del sitio) y el alto tiempo que las y los visitantes se detenían en este hito. En términos cualitativos, además, la Torre concentraba, según los resultados de aquella investigación, las principales emociones que po-

\footnotetext{
${ }^{15}$ El enfoque teórico que promueve el Área Museo del Parque por la Paz Villa Grimaldi es el de la "museología crítica", a través del cual, sobre principios constructivistas, se busca que las y los visitantes se apropien de la información y objetos dispuestos, generando interrogantes y reflexiones propias sobre la base de sus memorias (Aguilera, 2011a, p. 69).

${ }^{16}$ El estudio de público del Parque por la Paz Villa Grimaldi es un documento de uso interno. Sin embargo, los autores, en tanto integrantes del Área Museo de esta institución, pueden facilitar los resultados completos de la investigación a quienes lo soliciten, previa evaluación del requerimiento.
} 
dían ser consideradas como "negativas" (miedo, rabia y dolor) en relación a la experiencia generada ${ }^{17}$.

\section{CRECIENTE INTERÉS POR CONOCER MÁS SOBRE LOS TORTURADORES}

Estudios más recientes revelan el interés por parte del público del PPVG por conocer más sobre los torturadores ${ }^{18}$. En las encuestas realizadas durante el Día del Patrimonio Cultural entre los años 2010 y 2018, frente a la pregunta "¿Qué temas o información le interesaría encontrar en este lugar?", la opción acerca de obtener más antecedentes sobre los represores obtuvo un promedio de $15 \%$, posicionándose en segundo lugar de preferencia, empatando con "Violaciones a los DDHH en el lugar", por encima de alternativas como "Los derechos humanos hoy en Chile, en situaciones actuales" y "Causas del Golpe del Estado".

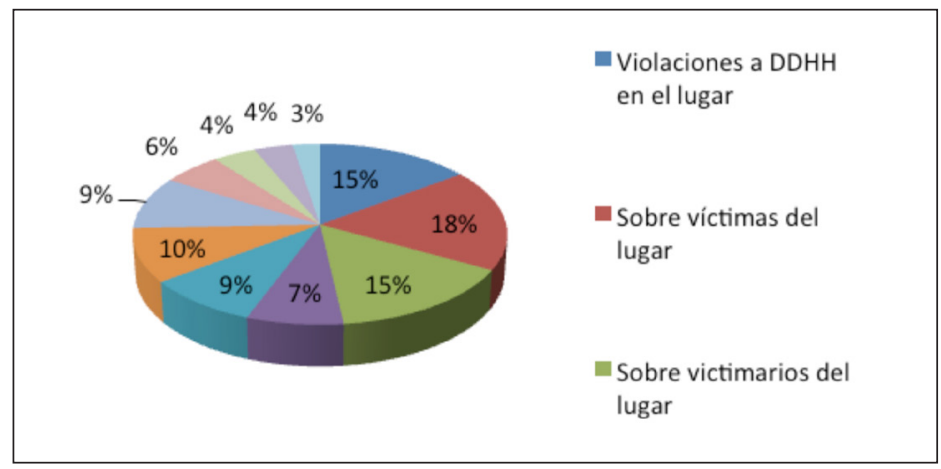

Figura 3. Promedio del período 2010-2018 de temas o informaciones que visitantes quisieran encontrar en el museo de sitio Villa Grimaldi. Fuente: Elaboración propia a partir de encuestas realizadas el Día del Patrimonio Cultural entre los años 2010 y 2018.

\footnotetext{
${ }^{17}$ Si bien la Celda presentó una frecuencia similar, esta es también relacionada con sensaciones "positivas", como la "solidaridad" y la "empatía", debido a la presencia de testimonios que hablan de muestras humanidad entre las/os detenidas/os que compartían prisión.

${ }^{18}$ Estos estudios se refieren a la interpretación de las encuestas realizadas anualmente a los visitantes durante el Día del Patrimonio Cultural por la Corporación Parque por la Paz Villa Grimaldi. Si bien se trata de documentos de uso interno, los autores, en tanto integrantes del Área Museo de esta institución, pueden facilitar los resultados completos de la investigación a quienes lo soliciten, previa evaluación del requerimiento.
} 
El incremento del interés del público por obtener mayores detalles sobre los victimarios ha sido sostenido durante los ocho años de aplicación de este instrumento. En el período 2011-2013, es notorio el mayor interés expresado sobre esta alternativa, en comparación con los años comprendidos entre 2015 y 2017. Una primera lectura preliminar de estos datos, podría relacionar el interés por obtener más antecedentes sobre los torturadores con la existencia de un gobierno de derecha, considerado los años del primer gobierno de Sebastián Piñera (2010-2013) y el alza experimentada en la encuesta de 2018, el primer año de su segundo mandato, en tanto en estos períodos se han generado una serie de hechos jurídicos e históricopolíticos relacionados con los victimarios y la enseñanza y conmemoración del pasado represivo ${ }^{19}$.

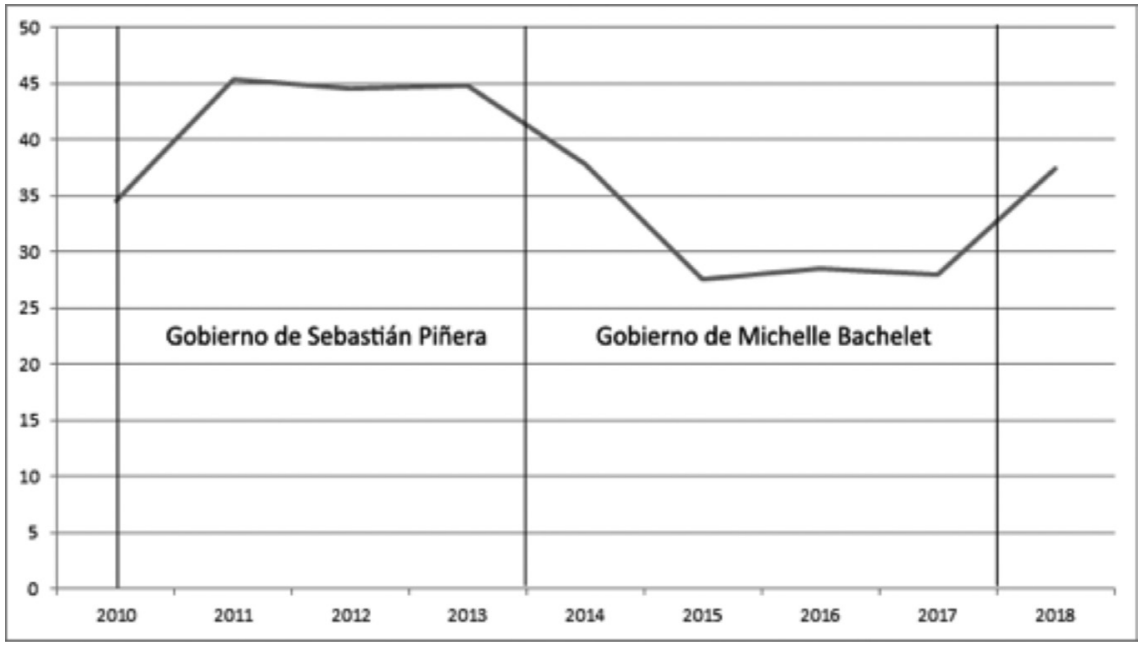

Figura 4. Evolución del porcentaje de menciones relacionadas al interés de información con respecto a victimarios de Villa Grimaldi. Fuente: Elaboración propia a partir de encuestas realizadas el Día del Patrimonio Cultural entre los años 2010 y 2018.

\footnotetext{
${ }^{19}$ Algunos de estos hechos fueron: el debate social generado en 2012 por la intención del Ministerio de Educación de modificar los textos de la asignatura de Historia, reemplazando el término "dictadura" por el de "gobierno militar"; la cuadragésima conmemoración en 2013 del golpe de Estado, contexto en el cual, el Presidente Piñera instaló en el escenario público la idea de los "cómplices pasivos" y; el cierre del Penal Cordillera en septiembre de 2013.
} 
En segundo lugar, otra interpretación de este fenómeno de incremento del interés por los perpetradores se puede relacionar con el cambio generacional que el público del PPVG está experimentando. En los últimos tres años, el segmento de visitantes más jóvenes, es decir, aquellos que tienen entre 0 y 19 años, pasó de representar un tercio del total del público general, a superar el 50\%. Para el Parque por la Paz, esta es una dimensión clave, entendiendo que su principal público corresponde a estudiantes secundarios, quienes, en su mayoría, se acercan por primera vez al estudio del pasado dictatorial desde la voz de las víctimas ${ }^{20}$.

Por último, un antecedente importante a destacar en este análisis es que no existe una relación directa entre mayor interés por los represores y un más alto deseo por encontrar museografía relacionada con la tortura ${ }^{21}$.

\section{REFLEXIONES FINALES}

Abordar las oportunidades de representación de represores en el PPVG, implica reconocer dos hechos. Primero, que el actual sitio de memoria surge de la urgencia de recuperar y poner en valor un ex CDTE que estaba demolido e invisibilizado, siendo reflejo de un proceso transicional que no desarrolló una política de memoria sobre estos lugares. Su reconstrucción como Parque y la discusión previa acerca de su constitución, expresan las tensiones y visiones de aquellos años. Segundo, considerar que el principal eje del proceso transformativo del sitio fue la resignificación, reemplazando "muerte por vida, olvido por memoria, silencio por testimonio, clandestino por público" (Raggio, 2011, p. 74), y propiedad de victimarios por lugar para las víctimas. Por lo tanto, la "muerte" y sus agentes no debían figurar entre los elementos configurativos del relato oficial.

En lo relativo a la incorporación práctica de victimarios a la narrativa y la museografía del PPVG, es posible evidenciar significaciones en dos sentidos. Por un lado, sobre las explicaciones del terrorismo de Estado ejercido a nivel nacional y, particularmente, en Villa Grimaldi, al distinguir la composición del aparato represivo, identificando la diferencia entre agentes (militares y civiles) y guardias. Por otro lado, se ha contribuido a mejorar el

\footnotetext{
${ }^{20}$ Para profundizar en esta materia, se recomienda revisar Veneros y Toledo (2009).

${ }^{21}$ En las encuestas realizadas durante el Día del Patrimonio, también se consulta a los visitantes acerca de qué elementos les interesaría encontrar en el sitio como apoyo a la narrativa. La opción "Elementos de tortura y apremio" ha descendido del $31 \%$ de las preferencias al 15\%, en el período 2010 2018.
} 
"qué y cómo contar" la historia del lugar al permitir profundizar, desde una perspectiva pedagógica, la narrativa en materias como la justicia transicional, los pactos de silencio y el comportamiento de los agentes al interior del recinto. Todos estos asuntos se enfrentan, actualmente, a un interesante desafío en términos de la representación museográfica del sitio y la efectividad del guion educativo: el creciente interés de las audiencias, especialmente, de las nuevas generaciones que no vivieron los procesos históricos abordados, por conocer más información acerca de los perpetradores. En este sentido, es probable que al interior del PPVG se genere una discusión acerca de su función social en el presente.

Por último, es necesario señalar que el proceso seguido por el Parque por la Paz en esta materia no está concluido. Reconociendo el valor de los avances expuestos, es ineludible destacar que existen asuntos relacionados con la representación de represores que deben ser trabajados y que, por su naturaleza, comprenderán nuevos nudos de memorias, como, por ejemplo, el futuro debate por la eventual inclusión de testimonios de ex guardias del recinto en el Archivo Oral. En este sentido, el proyecto Museo que se desarrolló en el lugar aún comprende oportunidades para la representación del terrorismo de Estado de manera integral, complejizando el uso reparatorio del sitio, incorporando elementos y soportes que permitieran proyectar la función educativa del lugar y, en definitiva, encausando la definición del PPVG como un sitio de conciencia. En este proceso, la representación de perpetradores puede ser una oportunidad para abordar las conflictividades de la memoria nacional respecto de su pasado reciente, promoviendo reflexiones críticas entre los visitantes.

\section{REFERENCIAS}

Aguilera, C. (2011a). Hacia una perspectiva de Educación en Derechos Humanos a partir de la experiencia de Villa Grimaldi. En Aguilera, C. y Millán, R. (eds.). Ciudadanía y Memoria. Desarrollo de Sitios de Conciencia para el aprendizaje en derechos humanos (pp. 55-72). Santiago, Chile: Corporación Parque por la Paz Villa Grimaldi.

Aguilera, C. (2011b). Proyecto de Museo en Villa Grimaldi. Una apuesta participativa de construcción. En Aguilera, C. y Cárcamo, C. (eds.). Ciudad y memorias. Desarrollo de sitios de conciencia en el Chile actual (pp. 100-109). Santiago, Chile: Corporación Parque por la Paz Villa Grimaldi.

Aguilera, C. (2019). Patrimonio hostil y pasado reciente. Londres 38. En Márquez, F. (ed.). Patrimonio. Contranarrativas Urbanas (pp. 93-114). Santiago, Chile: Universidad Alberto Hurtado. 
Alegría, L. (2010). Trayectoria educativa en un sitio de memoria: Corporación Parque por la Paz Villa Grimaldi. En Hurtado, J., Lamas, V., Matus, V., Walch, R. (eds.). Pedagogía de la memoria. Desafío para la Educación en Derechos Humanos (pp. 88-100). Santiago, Chile: Heinrich Böll Cono Sur.

Alegría, L. (2011). Patrimonio y atrocidad. En Marsal, D. (comp.). Hecho en Chile. Reflexiones en torno al patrimonio cultural (pp. 293-320). Santiago, Chile: Marval.

Archivo Oral, Corporación Parque por la Paz Villa Grimaldi.

Basaure, M. (2018). La monstruosidad y Pinochet. El Desconcierto [online]. Disponible en: https://www.eldesconcierto.cl/2018/05/24/la-monstruosidad-y-pinochet/

Bustamante, N. (2007). Los "pelaos" y las armas. La visión de los conscriptos del Ejército chileno. sobre el Chile de 1970-1975 durante y después de su servicio militar. Tesis para optar al grado de Licenciado en Historia. Santiago, Chile: Universidad de Chile.

Calveiro, P. (2006). Poder y desaparición. Los campos de concentración en Argentina. Buenos Aires, Argentina: Colihue.

Collins, C. (2013). Chile a más de dos décadas de justicia de transición. Política LI(2), 79-113.

Dalla Porta, C., Ayress, R., Salinas, A. (2017). 20 años Sitio de Memoria Parque por la Paz Villa Grimaldi. Santiago, Chile: Consejo Nacional de la Cultura y las Artes; Corporación Parque por la Paz Villa Grimaldi.

Elgueta, G. (2018). Institucionalización y patrimonialización de sitios de memoria en Chile. Una lectura desde la experiencia de Londres 38. Revista Aletheia VIII(16). Disponible en: https://www.aletheia.fahce.unlp.edu.ar/ article/view/ATHv8n16a11

Estefane, A. (2019). Pinochet y los "Hijos de la Libertad". Contornos de una polémica respecto del pasado reciente. En Estefane, A., Olmedo, C. y Thielemann, L. (eds.). 1988-1968: de la transición al largo '68 en Chile (pp. 275277). Santiago, Chile: Ariadna.

Gryglewski, E. (2017). ¿Por qué investigar sobre violadores de derechos humanos y autores de crímenes de lesa humanidad? En Jara, D. y Aguilera, C. (eds.). Pasados Inquietos (pp. 16-33). Santiago, Chile: Museo de la Memoria y los Derechos Humanos; Foro Urbano.

Jara, D. y Aguilera, C. (2017). Pasados inquietos. Dilemas en torno al lugar de los perpetradores en las sociedades postconflicto. En Jara, D. y Aguilera, C. (eds.). Pasados inquietos (pp. 8-15). Santiago, Chile: Museo de la Memoria y los Derechos Humanos; Foro Urbano.

Lazzara, M. (2003). Tres recorridos de Villa Grimaldi. En Jelin, E. y Langland, V. (comps.). Monumentos, memoriales y marcas territoriales (pp. 127-148). Madrid, España: Siglo XXI.

Mallea, F. y Meirovich, S. (2019). ¿Por qué (no) se puede incluir a Pinochet en una muestra museográfica? En Estefane, A., Olmedo, C. y Thielemann, L. 
(eds.). 1988-1968: de la transición al largo '68 en Chile (pp. 284-286). Santiago, Chile: Ariadna.

Mallol, C. (2009). Renacer en la agonía. De la sobrevida a la vida. Estudios Públicos (115), 31-48.

Marchesi, A. (2018). Memoria ejemplar y literal. En Vinyes, R. (dir.). Diccionario de la memoria colectiva (pp. 284-285). Barcelona, España: Gedisa.

Raggio, S. (2011). La relación pasado-presente en las propuestas educativas de los "sitios de memoria". En Aguilera, C. y Millán, R. (eds.). Ciudadanía y Memoria. Desarrollo de Sitios de Conciencia para el aprendizaje en derechos humanos (pp. 73-80). Santiago, Chile: Corporación Parque por la Paz Villa Grimaldi.

Romero, M. (2011). Apertura. En Aguilera, C. y Millán, R. (eds.). Ciudadanía y Memoria. Desarrollo de Sitios de Conciencia para el aprendizaje en derechos humanos (pp. 13-14). Santiago, Chile: Corporación Parque por la Paz Villa Grimaldi.

Salazar, G. (2013). Villa Grimaldi (Cuartel Terranova). Historia, testimonio, reflexión, 1a edic. Santiago, Chile: Lom.

Salvi, V. (2016). Los represores como objeto de estudio. Cuadernos del IDES $32,22-41$.

Salvi, V. (2017). El universo de los represores: una mirada desde los estudios de memoria en la Argentina. En Jara, D. y Aguilera, C. (eds.). Pasados Inquietos (pp. 34-56). Santiago, Chile: Museo de la Memoria y los Derechos Humanos; Foro Urbano.

Santos-Herceg, J. (2016a). Los centros de detención y/o tortura en Chile. Su desaparición como destino. Revista Izquierdas 26, 256-275.

Santos-Herceg, J. (2016b). Geografía humana del horror. Agentes, detenidos y transeúntes. En Pizarro, C. y Santos-Herceg, J. (comps.). Revisitar la catástrofe. Prisión política en el Chile dictatorial (pp. 167-186). Santiago, Chile: Pehuén.

Seguel, P. (2017). La dimensión política y social de los procesos de puesta en valor del patrimonio de la memoria de las violaciones a los derechos humanos en Chile. 1996-2016. En Cabeza, A., Cárdenas, A., Lawner, M., Seguel, P. y Bustamante, P. Patrimonio de la memoria de los derechos humanos en Chile (pp. 25-35). Santiago, Chile: DIBAM; Consejo de Monumentos Nacionales.

Sepúlveda, V. (2016). Semblanzas del torturador pinochetista. En Pizarro, C. y Santos-Herceg, J. (comps.). Revisitar la catástrofe. Prisión política en el Chile dictatorial (pp. 189-204). Santiago, Chile: Pehuén.

Stern, S. (1999). De la memoria suelta a la memoria emblemática: hacia el recordar y el olvidar como proceso histórico (Chile, 1973-1998). En Garcés, M., Milos, P., Olguín, M., Pinto, J., Rojas, M.T. y Urrutia, M. (comps.). Memoria para un nuevo siglo. Chile, miradas a la segunda mitad del siglo $X X$ (pp. 11-33). Santiago, Chile: Lom. 
Tunbridge, J. y Ashworth, G. (1996). Dissonant Heritage: The Management of the Past as a Resource in Conflict. New York, United States: J. Wiley.

Veneros, D. y Toledo, M. (2009). Del uso pedagógico de lugares de memoria: visita de estudiantes de educación media al Parque por la Paz Villa Grimaldi (Santiago, Chile). Revista Estudios Pedagógicos XXXV(1), 199-220. 